\title{
AN APPLICATION OF COMPUTER CONFERENCING IN DENTAL EDUCATION
}

\author{
W. PAUL LANG
}

School of Dentistry, University of Michigan, Ann Arbor, MI 48109-1078 (U.S.A.)

(Received May 29th, 1992)

(Accepted June 8th, 1992)

\begin{abstract}
A computer conference was used as an instructional tool in a dental school course on health education. Second year students $(N=84)$ were divided into 17 groups to design community education programs. A student from each group participated in the conference to electronically discuss their program with peers. The conference operated for 52 days. The 28 conference topics were displayed 1028 times. There were 279 conferencing uses lasting $8125 \mathrm{~min}$ (135.4 h); a mean of $29.1 \mathrm{~min} /$ use. Mean responses per students was 25 . Mean student terminal time was $7.8 \mathrm{~h}$. Instructor terminal time was $20.8 \mathrm{~h} ; 5.4 \mathrm{~h}$ were spent managing the conference. Of the available conferencing funds $(\$ 440.00)$, $\$ 218.35$ was expended with students using \$168.24. Mean dollars spent per student was \$9.90. Quantitative evaluation of participation was based on: (a) number of individual responses made compared to mean responses; (b) timely item placement on the conference; and (c) the percentage of responses made in other students' items. Student evaluation of conferencing was positive and acknowledged the value of computer skills.
\end{abstract}

Key words: Dental education; Computer communication networks; Computer systems

\section{Introduction}

The utility of computer conferencing, a rapidly growing communication medium, can be observed by its applications in dentistry. A dental public health conference - Dent:Forum - has functioned since 1985 [1,2]. Besides providing information on profession-specific topics, this conference has been used to post employment and training opportunities and announce professional meetings. Other conference uses have been suggested including communicating with colleagues and publishing dental society newsletters [3]. The advantages of conferencing include group discussion unhindered by time and locality differences, access to information from remote sites and electronic messaging [2,4].

Educational applications of conferencing have received limited attention in dentistry although their use in other settings has been evolving [5]. Computer conferences or 'Electronic Classrooms' at Indiana University provide instructors with additional opportunities for discussions with students, allow students to express their opinions and encourage a sense of community among participants [6]. Also,

Correspondence to: Dr. Lang, Department of Periodontics/Prevention and Geriatrics School of Dentistry, University of Michigan Ann Arbor, Michigan 48109-1078, U.S.A. 
students are sensitized to the positive and negative aspects of the technology. Educational conferences encourage participant-specific questions and answers, facilitate easy but leisurely responses and help develop critical thinking and communication skills [7]. More mundane uses of these conferences include distribution and submission of assignments and class announcements. Generally, computer-mediated communication has been found to be as effective as traditional face-to-face education but improved outcomes are dependent upon faculty skill with the medium, student motivation and accessibility of equipment [8].

Thus, computer conferencing can potentially assume dual roles as a means of information access and as an adjunct to instruction. The value of information access and telecommunications has been recognized by medicine and dentistry [9-11], but medicine has articulated that value into educational competencies for its students. Computer conferencing fits a model proposed by Lindberg for developing training and competencies in informatics in medical education [12]. Conferencing addresses two levels of understanding in Lindberg's taxonomy of competencies: level three, minimal personal skills and level four, the knowledgeable consumer. These levels have been developed further by an informatics task force at the University of Maryland [13]. According to the task force, minimal personal skills include using computer systems, accessing databases, evaluating existing systems and understanding hardware and software concepts. The knowledgeable consumer should be able to use specialized systems and databases and evaluate and make informed decisions about information systems particular to the individual's field.

The Maryland model can be applied to dentistry and minimal personal computer skills and dental informatics applications to create knowledgeable consumers have been proposed [14]. While not specified, computer conferencing can be considered an application component of dental informatics that is supported by the American Association of Dental Schools (AADS) for incorporation into dental curricula [15]. A conference on strategic issues for dental informatics also encouraged use of computers and related tools to integrate informatics into the instructional process [16]. For dentistry, computer conferencing can be used to support instruction and as a means of information access with the latter's being more likely to develop in dental practice as information management becomes a professional imperative. Given that solo practice is still the predominant mode in dentistry, developing access and retrieval skills during dental school is critical so that once in practice, graduates can take advantage of remote information resources and avert isolation by developing 'connectivity.'

This paper describes the use of computer conferencing in a dental health education course for second year dental students. The goals of the project were to: (1) use computer conferencing to support instruction; (2) introduce dental students to conferencing for information access; and (3) assess students' use and perceived value of conferencing.

\section{Methods}

A health education course was designed to have second year students $(N=84)$ plan community dental health education programs in randomly selected groups. All 
groups $(N=17)$ were given a dental topic intended for a specific community organization or population. Within each group, individuals self-selected tasks for constructing the presentation. One member became the leader to direct the group; another member developed a program plan. A third member performed a MEDLINE search to identify educational content for the presentation. Another member participated in a computer conference to discuss the intended program with peers. A fifth member constructed an evaluation instrument to be used to assess program outcomes. A final report that combined all the elements was submitted to the instructor. Groups were randomly selected to present their program plans in two class sessions.

The students accessed the conference in the dental school's CAIDENT (Computer-Assisted Instruction, Dentistry) Center which contains 34 Macintosh SEs with 50 megabyte hard drives. VersaTerm, version 4.1.1 was used to communicate with the Michigan Terminal System and the campus mainframe computer. The total number of hours available for student access to CAIDENT was $77.5 \mathrm{~h}$ including evenings and weekends. The conference could also be accessed remotely using a computer and modem.

The conferencing program was CONFER II [17]. A written permanent record is created as the conference develops and summary statistics are compiled. An accounting management program is used to dispense computing funds and track accounts. Funds for conferencing - $\$ 440.00$ - were acquired from the University's General Fund Computing Allotment (GFCA). The GFCA supports unsponsored research, educational activities, thesis research and request accounts for personal computing. Student accounts were assigned as part of the class rather than as personal request accounts. The conference (HealthEd) resided in a computer account accessible to participants but with management access restricted to the instructor. The instructor (organizer) used another account to participate while each student was given a separate account. Students were allotted $\$ 10.00$ initially and use was monitored by the instructor who could add money to accounts as necessary. Two guest accounts were also available but not used. Two more individuals actively participated in the conference using personal accounts. One was a professor emeritus of public health dentistry who accessed the conference from his home; the other was a faculty member from another dental school.

To introduce conferencing, an overview of the technic was given during the first lecture. The use of communications software was described along with sign-on procedures for accessing the conference through the university terminal system. Conference methods were displayed such as producing a conference discussion item, displaying an item, responding to an item and retrieving messages within the conference. A dental public health conference $[1,2]$ was described as an example of a conference. The 17 students who elected to participate in the conference were also given the option of attending a 1.5-h workshop on CONFER offered by the University's computer center. Students were then required to sign on the conference, place at least one item related to their program topic, respond to other items and participate for the duration of the course.

The conference was organized around items that were classified broadly into three groups: conference and course information, health education topics and 
miscellaneous. For example, in conference information, items included commands to help control the computer, computer etiquette and university policy on direct harassment. In course information, directions were given for submitting papers and the format of the final report was described. The health education items were selected to represent topical issues that dentists might need to address in their practices based on patient inquiries or community requests for education. These items included mercury in dental amalgam, infection control, fluoride and cancer, smokeless tobacco, fissure sealants and oral hygiene instruction for various groups. The third category - miscellaneous - was placed as an item where students could talk about anything they considered of interest. Topics included an extra residency year for dental students, educational emphasis on technical aspects of dentistry and the business of dentistry.

Evaluation of student participation was based on quantitative measures and included the number of responses made per student compared to the mean number of responses, whether students placed their items on the conference in a timely fashion and the percentage of responses made in other students' items rather than in the students' own items. Item placement was considered timely if performed by the second week of class. As part of this investigation, qualitative response analyses were performed on three items with high, medium and low numbers of responses by categorizing item responses as informational responses, instructor responses, questions, or other responses. Students evaluated the conferencing experience by responding to 18 close-ended questions having a 5-point Likert scale ranging from Strongly Agree -1 to Strongly Disagree -5 and to two open-ended questions on likes and dislikes of conferencing.

\section{Results}

The conference operated for 52 days. Summary statistics are shown in Table I. Twenty persons participated and there were 28 items placed on the conference that were displayed a total of 1028 times. A total of 279 uses occurred with $8125 \mathrm{~min}$ $(135.4 \mathrm{~h})$ of conference use. The mean minutes per use was 29.1 . Ninety-one private

\section{TABLE I}

SUMMARY STATISTICS FOR THE HEALTHED CONFERENCE

\begin{tabular}{lr}
\hline Characteristic & $(N)$ \\
\hline Number of response items & 28 \\
Number of participants & 20 \\
Number of uses & 279 \\
Minutes of use & 8125 \\
Private messages sent & 91 \\
Number of times items displayed & 1028 \\
Number of responses made & 646 \\
Sets of responses seen & 2148 \\
Number of commands issued & 1939 \\
\hline
\end{tabular}


messages were sent among participants. Participant responses totaled 646. Table II displays the conference items by frequency of response. While topical items like mercury in dental amalgam and infection control were frequently discussed, other items like bite splints and mouthguards received attention. All but three students placed their items on the conference by the end of the second week of class - one of the grading criteria.

Item analysis was performed on three items with high, medium and low numbers of responses. Bite splints was the high response item; periodontal disease prevention was the medium response item; and alcohol, tobacco and oral cancer was the low response item. The analysis is shown in Table III. The most common response provided information and these responses accounted for $46-69 \%$ of total responses across the three items. Instructor responses comprised $16-24 \%$ of responses, while responses that asked a question accounted for $0-17 \%$ of responses. Other responses accounted for $7-21 \%$ of responses across the three items.

TABLE II

CONFERENCE ITEMS AND NUMBER OF RESPONSES BY TOPIC

\begin{tabular}{ll}
\hline No. of Conference items & No. of responses \\
\hline Conference and course information & 53 \\
Welcome iutems & 18 \\
Course information & 12 \\
Ground rules & 11 \\
Editing tips & 1 \\
MTS and CONFER HELP & \\
& \\
Dental health education items & 48 \\
Bite splints & 47 \\
Mouthguards & 45 \\
Mercury in dental amalgam & 45 \\
Infection control & 36 \\
Root caries in older adults & 34 \\
Proper use of fissure sealants in children & 30 \\
Baby bottle tooth decay & 29 \\
Prevention of periodontal disease in adults & 29 \\
Fluoride use and cancer & 28 \\
Anti-calculus toothpastes & 26 \\
Oral hygiene instruction for older adults & 26 \\
Brushing/flossing recommendations for children & 23 \\
Care of removable dental appliances & 21 \\
Fractured incisors in grade school children & 21 \\
Oral hygiene for developmentally disabled children & 20 \\
Hazards of smokeless tobacco & 19 \\
Alcohol, tobacco and oral cancer & \\
Miscellaneous & 24 \\
New policy for dental students (Possibility of a residency year) & \\
\hline
\end{tabular}


TABLE III

ITEM RESPONSE TYPE BY RESPONSE FREQUENCY

\begin{tabular}{lccc}
\hline Response type & $\begin{array}{l}\text { High } \\
(N=48)\end{array}$ & $\begin{array}{c}\text { Medium } \\
(N=29)\end{array}$ & $\begin{array}{c}\text { Low } \\
(N=19)\end{array}$ \\
\hline Informational & $46 \%$ & $69 \%$ & $58 \%$ \\
Instructor & $22 \%$ & $24 \%$ & $16 \%$ \\
Questions & $17 \%$ & $0 \%$ & $5 \%$ \\
Other & $15 \%$ & $7 \%$ & $21 \%$ \\
& & & \\
Total & $100 \%$ & $100 \%$ & $100 \%$ \\
\hline
\end{tabular}

The cost and terminal time per user are displayed in Table IV. The total number of dollars allocated does not include the two unused guest accounts $(\$ 20.00)$. The conference management account expended about $\$ 25.00$ while the instructor used an equivalent amount to participate. Only five students exceeded the initial account deposit of $\$ 10.00$. Of the $\$ 440.00$ available for total conference use, $\$ 218.35$ was

\section{TABLE IV}

EXPENDITURES, TERMINAL TIME, AND PERSONAL RESPONSE ANALYSIS BY PARTICIPANT

\begin{tabular}{llllll}
\hline Participant & $\begin{array}{l}\text { Dollars } \\
\text { allocated }\end{array}$ & $\begin{array}{l}\text { Dollars } \\
\text { spent }\end{array}$ & $\begin{array}{l}\text { Terminal } \\
\text { time (h) }\end{array}$ & $\begin{array}{l}\text { No of } \\
\text { Responses }\end{array}$ & $\begin{array}{l}\text { \% of Student } \\
\text { responses } \\
\text { in own item }\end{array}$ \\
\hline Conference & 85.00 & 24.69 & 5.4 & - & - \\
Instructor & 60.00 & 25.42 & 15.4 & 165 & NA $^{\mathrm{a}}$ \\
Student A & 25.00 & 20.64 & 19.1 & 48 & 50 \\
Student B & 20.00 & 15.88 & 11.3 & 64 & 32 \\
Student C & 20.00 & 15.47 & 13.4 & 30 & 42 \\
Student D & 20.00 & 13.04 & 10.2 & 28 & 16 \\
Student E & 20.00 & 12.56 & 10.1 & 31 & 35 \\
Student F & 15.00 & 9.26 & 8.1 & 29 & 24 \\
Student G & 15.00 & 9.08 & 6.2 & 20 & 32 \\
Student H & 15.00 & 9.03 & 7.4 & 22 & 28 \\
Student I & 15.00 & 8.78 & 7.3 & 18 & 16 \\
Student J & 15.00 & 8.61 & 7.6 & 15 & 42 \\
Student K & 10.00 & 7.74 & 6.2 & 21 & 21 \\
Student L & 15.00 & 6.72 & 4.1 & 12 & 14 \\
Student M & 15.00 & 6.66 & 5.2 & 10 & 13 \\
Student N & 15.00 & 6.55 & 4.3 & 29 & 19 \\
Student O & 15.00 & 6.23 & 5.2 & 9 & 29 \\
Student P & 15.00 & 6.14 & 3.4 & 21 & 14 \\
Student Q & 10.00 & 5.85 & 3.5 & 18 & 15 \\
& & & & & \\
Other faculty & NA & NA & NA & 56 & NA \\
& & & & & 26 \\
Totals & 420.00 & 218.35 & 153.4 & 646 & \\
\hline Not applicable. & & & & & \\
\hline
\end{tabular}

${ }^{a}$ Not applicable. 
expended. Students used $\$ 168.24$. The mean number of dollars spent per student account was $\$ 9.90$. The effects of the University's variable rate structure can be observed in the dollars spent per terminal times of Students $G$ and $\mathbf{H}$. Student $\mathbf{H}$ signed on the computer at more economical times of the day than Student $G$ with the result's being more than an hour of additional terminal time consumed for about the same amount of dollars spent.

The total amount of terminal time was $153.4 \mathrm{~h}$. The disparity between this figure and total conference use $(135.4 \mathrm{~h})$ is due to conference management time $(5.4 \mathrm{~h})$ and time spent by participants on their accounts but not on the conference $(12.6 \mathrm{~h})$. Student terminal time ranged from 3.4 to $19.1 \mathrm{~h}$ with the mean being $7.8 \mathrm{~h}$. The number of responses per student varied from 9 to 64 with the mean being 25 responses. For all students, about one-quarter of their responses were in their own item. The greatest percentage of own-item responses was $50 \%$.

Table V displays the questions used to evaluate the conference experience. Four

\section{TABLE V}

MEAN EVALUATION SCORE BY QUESTION $(N=17)$

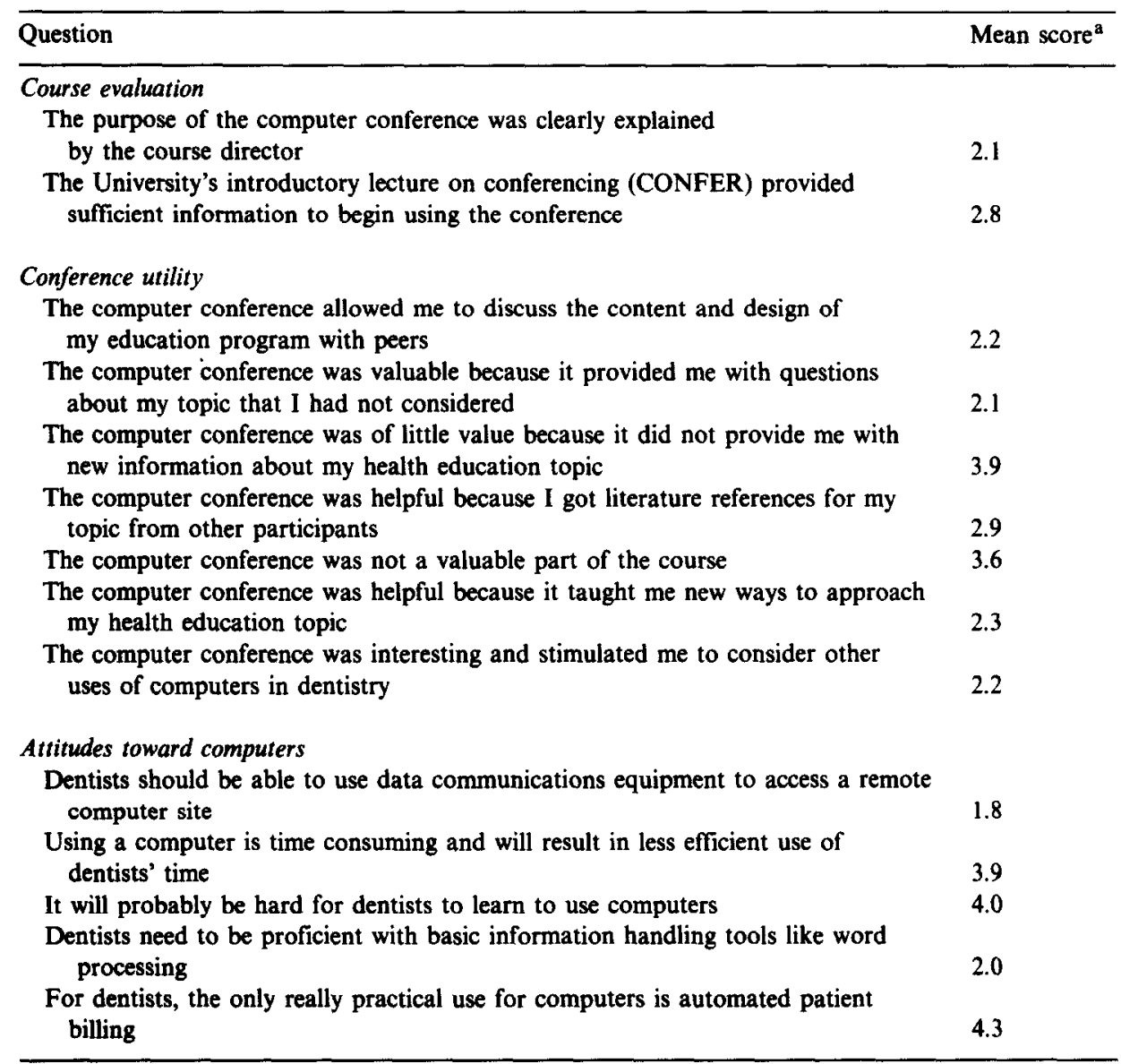

${ }^{\text {a Strongly agree }}=1$, Undecided $=3$, Strongly disagree $=5$. 
questions relating to instructor evaluation are not displayed. The students agreed that the purpose of the conference was clearly explained but were undecided about the sufficiency of the introductory lecture provided by the University. Students' perceptions of conference utility were generally positive but more neutral responses were recorded for the helpfulness of receiving literature references from other participants. Students thought they received new information about their topic through the conference but were less certain about the value of conferencing as part of the course. Attitudes of the students toward computer use by dentists were positive. Students felt it would not be hard for dentists to learn to use computers nor that the only practical use for computers was patient billing.

\section{Discussion}

Successful computer conferencing requires that the organizer is actively involved, acquires training, sets goals, clarifies expectations of participants and establishes norms for behavior on the conference [7]. For HealthEd, these requirements were mostly met and some additional observations can be made about managing a conference. For instructional purposes, the length of time the conference operated was optimal as it was short enough to keep students' interest yet long enough to generate discussion on the topics. If the conference time were to be extended, more items or more participants would probably be needed.

The addition of more items could help the conference or possibly dilute its effect if its purpose was very specific. It has been suggested that 'fun' or 'outside' items should be placed on a conference to keep interest [7]. On HealthEd, most discussion centered on the education topics with little conversation about unrelated topics. In the future, a few non-course-related items may be included and response frequencies observed. The addition of more student participants is possible but would add to conference management time. Ideally, all students should experience electronic communication and conferencing but this experience may have to occur incrementally in a curriculum. The participation of other faculty on the conference was invaluable as it brought fresh views and experiences to the conference.

The mean minutes per use was higher than expected and perhaps reflects the fact that student participants were conferencing novices. The number of responses per item is indicative of audience interest in a topic and the ability of the item owner to stimulate conversation about the topic. For example, bite splints was a popular topic because many participants had had personal experiences with these dental appliances. Some of the less discussed items suffered from student inexperience with the topic or less interest in the subject. Analysis of the three items indicated that informational responses generally comprised a high percentage of total responses. In two items, instructor responses accounted for nearly one-quarter of all responses. The instructor can expect, at least initially, to be responsible for propelling the conference and participating frequently. The extent of instructor participation will depend on student willingness to talk on the conference, their knowledge of a subject and their confidence in using the system.

The cost of operating the conference was within its budget as only half the available funds were spent. Students were not excessive in their use of terminal time and even the most frequent user expended only $\$ 21.00$. The time spent by students 
on the system ranged widely and can be seen to be related somewhat to numbers of responses. These variables can be affected, however, by the conferencing skills of the operator, the length of responses and the time spent on the system but out of the conference. For example, the instructor made 165 responses and used $15.4 \mathrm{~h}$ of time which reflect expertise and frequent short responses. Conversely, one student made only 9 responses during $5.2 \mathrm{~h}$ of terminal time. In the context of the course, this student's participation was less than optimal given expectations for student involvement. In a functioning 'real world' computer conference, this individual would be considered an observer rather than an active participant and observation can be valuable. The conference mode, it is hoped, will stimulate reticent students to participate as has been suggested [2], but non-use may be related to lack of interest in the activity. There is also the danger of excessive participation although students did not seem to have this problem. Instructors must balance their participation between stimulating interactions and stifling them.

Penalizing students for responding more to their own items than others was problematic as some items needed more owner input than others. Conversely, it was clear that toward the end of the course, a few students were entering responses in their items merely to increase their participation level. These occurrences were infrequent and use of this grading criterion could be eliminated. A flurry of responses toward the end of the participation time should be expected. The problem of late item entry was minimal but could present problems for conference vitality. Explanation of conferencing expectations early in the course and periodic reiteration in the conference should alleviate these problems. The messaging system can also be used to reach individuals privately but they must have signed on the system and checked in regularly.

From an instructor's perspective, the conference was not difficult to manage and documentation was adequate [18]. About $20 \mathrm{~h}$ of instructor time were required for the exercise with the time's being expended in short segments. Instructor conferencing occurred primarily during the day but could easily have been accomplished after hours from a remote site. Conference interactions, interestingly, created good student-instructor rapport that has lasted beyond the course. Developing an evaluation scheme for conferencing was somewhat problematic and there were some student complaints about unknown expectations. A suggestion was made to have students critique each others' items and this peer review activity might be added to the evaluation scheme. Participation by more faculty would enhance the conference, but increase course overhead in expended faculty time.

The users viewed computer conferencing as having value and felt that they received new information about their topic from the conference. It is less clear what information was transferred. Program plans and results of MEDLINE searches were presented on the conference indicating one direction of information flow. What information transferred from the conference to the group is unknown. Clearly, conferencing and information acquisition require further investigation in educational settings and in dental practice. The students held positive attitudes towards other aspects of computer use in dentistry; a finding similar to previous investigations [19]. The conferencing experience did not appear to have alienated them from computer applications.

Perhaps the most important outcome and one that challenges measurement, is 
whether access and retrieval skills are adopted by students and carried into practice. The need for these skills is apparent. A dentist's ability to leave his/her practice and seek information is often restricted. Information sources within the practice such as textbooks or journals may not be current or need-specific. For solo practitioners, a spontaneous discussion with a colleague may not be possible. Perhaps the most persuasive argument for adopting electronic information access and retrieval technics is that they support professional growth and collegiality. Further, knowledge acquisition and collaboration become more dynamic as these events can occur at the convenience of the dentist. Linkages with centers of dental learning, third party insurers and other health professionals can be anticipated as appreciation for electronic connections increases. Computer conferences could be created for continuing education presentations, dental class communications following graduation, study clubs, or management software user groups. A computer, modem and communications software are the elements needed to make these connections.

In summary, computer conferencing was employed as an adjunct to instruction and as a demonstration of an electronic communication mechanism for dentists. Further evaluation of these functions is warranted to understand their potential and to promote their use in dental education and practice. Training students to use conferencing will create an expectation that hopefully can be fulfilled by the presence of this tool in the dental office.

\section{References}

1 Eklund SA: Is it time for CONFER, a computer-based conference for dental public health?, $J$ Pub Health Dent, 44 (1984) 73-77.

2 Weintraub JA and Eklund SA: Development of a computer-based communication network for a dental specialty group, $J$ Dent Educ, 52 (1988) 525-529.

3 Castaldo DA, Coates VH and Silber SA: The American dental network. Dentistry's information source, Dent Clin North Am, 30 (1986) 721-729.

4 Schneider SJ and Tooley J: Self-help computer conferencing, Comput Biomed Res, 19 (1986) 274-281.

5 Harasim L (Ed.): Online education: Perspectives on a new environment, Praeger, New York, 1990.

6 Hansen E, Brown B, Chong S, Kubota K, Totten N and Hubbard L: Computer Conferencing for Collaborative Learning in Large College Classes, Indiana University, 1991.

7 Consulting and Support Services: Information Technology Division. Confer II Support Packet, University of Michigan, Ann Arbor, 1990.

8 Hiltz SR: Evaluating the virtual classroom. In Online Education: Perspectives on a New Environment (Ed: L Harasim), Pergamon Press, Oxford, 1990, pp. 133-183.

9 Association of American Medical Colleges: Part II: The state-of-the-art in medical informatics. In Medical Education in the Information Age: Proceedings of the Symposium on Medical Informatics, Association of American Medical Colleges, Washington, DC, 1985, pp. 11-59.

10 Higgins MC and Newman AB: Information systems for office practice. In Medical Informatics. Computer Applications in Health Care (Eds: EH Shortliffe, LE Perreault, G Wiederhold and LM Fagan), Addison-Wesley Publishing Co, Reading, MA, 1990, pp. 400-433.

11 Stikeleather J, Hensel JS and Baumgarten SA: The computerized dental office of the future, Dent Clin N Am, 32 (1988) 173-190.

12 Lindberg DAB: Taxonomy of understanding of medical information. In Medical Education in the Information Age: Proceedings of the Symposium on Medical Informatics, Association of American Medical Colleges, Washington DC, 1986, pp. 92-94.

13 Ball MJ: Informatics in professional education. In International Symposium of Medical Informatics and Education (Eds: R Salamon, D Protti and J Moehr), University of Victoria, Victoria BC, 1989, pp. $435-440$. 
14 Lang WP and Ellis LB: Toward a curriculum in dental informatics, MegaBite, 1 (1990) 3-4.

15 Cameron CA: The AADS strategic plan for dental informatics, J Dent Educ, 54 (1990) 593-594.

16 Salley JJ, Zimmerman JL and Ball MJ (Eds.): Dental informatics: Strategic issues for the dental profession. In Lecture Notes in Medical Informatics (Eds: O Rienhoff and D Lindberg), Springer-Verlag, Berlin, 1990.

17 Advertel Communications Systems: The Beginner's Guide to Confer II., Advertel Communications Systems, Ann Arbor, 1981.

18 Advertel Communications Systems: The Organizer's Guide to Confer II., Advertel Communications Systems, Ann Arbor, 1981.

19 Lang WP, Green TG and Jacobson JJ: Students' knowledge, opinions and behaviors concerning dental informatics and computer applications, J Dent Educ, 52 (1992) 195-199. 